\title{
Role of electron scattering on the high-order harmonic generation from solids
}

\author{
Chang-Ming Wang, ${ }^{1}$ Nicolas Tancogne-Dejean $\odot,{ }^{1}$ Massimo Altarelli, ${ }^{1}$ Angel Rubio,,${ }^{1,}$ and Shunsuke A. Sato $\odot^{2,1, \dagger}$ \\ ${ }^{1}$ Max Planck Institute for the Structure and Dynamics of Matter, Luruper Chaussee 149, 22761 Hamburg, Germany \\ ${ }^{2}$ Center for Computational Sciences, University of Tsukuba, Tsukuba, Ibaraki 305-8577, Japan
}

(Received 20 April 2020; accepted 7 August 2020; published 28 August 2020)

\begin{abstract}
We extend the semiclassical trajectory description for the high-order harmonic generation (HHG) from solids by integrating the effect of electron-scattering. Comparing the extended semiclassical trajectory model with a one-dimensional quantum mechanical simulation, we find that the multiplateau feature of the HHG spectrum is formed by Umklapp scattering under the electron-hole acceleration dynamics by laser fields. Furthermore, by tracing the scattered trajectories in real-space, the model fairly describes the emitted photon energy and the emission timing of the HHG even in the higher plateau regions. We further consider the loss of trajectories by scattering processes with a mean-free-path approximation and evaluate the HHG cutoff energy as a function of laser wavelength. As a result, we find that the trajectory loss by scattering causes the wavelength independence of the HHG from solids.
\end{abstract}

DOI: 10.1103/PhysRevResearch.2.033333

\section{INTRODUCTION}

Light-matter interactions have been an important subject in physics from both fundamental and technological points of view [1-4]. Intense light may couple with matter nonlinearly and can induce nonlinear optical effects [5-9], such as the second-order harmonic generation [10]. Once field strength of light becomes extremely large, nonperturbative and highlynonlinear phenomena may be induced. A primary example of such phenomena is the high-order harmonic generation (HHG) [11-13], which is an extreme photon-upconversion process via strongly nonlinear light-matter interactions. This process has been observed from atomic gases decades ago $[14,15]$, and the gas-phase HHG further opened a novel technology to generate ultrashort laser pulses with attosecond duration, offering a novel avenue to explore ultrafast real-time electron dynamics in matter [16-21]. Recently, the HHG from solid-state materials has been systematically observed [22]. It has been demonstrated that the solid-state HHG shows distinct fundamental features from the gas-phase HHG, such as the linear scaling of the cutoff energy with respect to the field strength [22-24], the wavelength independence of the cutoff energy $[22,24,25]$, and the enhancement of HHG by elliptically polarized light [26,27]. It has also been shown that the competition of mechanisms between atomiclike and solidlike responses in two-dimensional systems further enriches the

\footnotetext{
*angel.rubio@mpsd.mpg.de

†ssato@ccs.tsukuba.ac.jp
}

Published by the American Physical Society under the terms of the Creative Commons Attribution 4.0 International license. Further distribution of this work must maintain attribution to the author(s) and the published article's title, journal citation, and DOI. Open access publication funded by the Max Planck Society.
HHG spectra [28-30]. These distinct features of the solid-state HHG have been drawing great attention because it offers a novel possibility to investigate ultrafast electron dynamics in matter and may open a path to novel light sources [31].

The mechanism of the HHG from gases has been understood by the semiclassical trajectory model; so-called threestep model $[13,32,33]$. The model consists of the following three steps: (i) An electron is ionized from an atom or molecule due to a strong laser field. (ii) The ionized electron is accelerated by the laser field in vacuum. (iii) The accelerated high-energy electron returns to the parent ion due to the oscillatory field and recombines, emitting high energy photons. The three-step model describes well the features of the gas-phase HHG such as the cutoff energy. The semiclassical trajectory model has been further extended to the solidstate HHG [34], integrating the electronic band dispersion of solids as the crystal-momentum-dependent effective mass of electron-hole pairs. In this regard, the extended semiclassical trajectory model still treats the dynamics of electron-hole pairs as that of free particles. However, in contrast to ionized electrons in vacuum, electrons in solids may be easily scattered by ions, other electrons, defects and so on. Therefore, electron scattering is expected to play an important role for the HHG from solids.

Despite the great effort to study the mechanism of the HHG from solids, the role of the electron scattering has not been investigated yet in the context of the semiclassical trajectory description. In this paper we consider an extension of the real-space semiclassical trajectory model by incorporating scattering effects in solids. The generalization is carried out by branching a classical trajectory into multiple trajectories whenever a scattering event occurs. We compare the scattering-integrated semiclassical trajectory model with one-dimensional quantum dynamical simulations, and explore a role of Umklapp scattering in the HHG from solids. Furthermore, we extend our modeling with 
the mean-free-path approximation and elucidate the effect of the scattering to the wavelength scaling of the $\mathrm{HHG}$ cutoff.

This paper is organized as follows: In Sec. II we first revisit the semiclassical trajectory model for HHG from solids. Then, we extend the model by incorporating the scattering effect. In Sec. III we examine the role of the scattering in the HHG with the model, comparing with the one-dimensional quantum model. Finally, our findings are summarized in Sec. IV. In this work, atomic units are used unless stated otherwise.

\section{METHODS}

\section{A. Semiclassical trajectory model for solid-state HHG}

Here, we first revisit a semiclassical trajectory model for the solid-state HHG [34] to further introduce the scattering effect into it. The semiclassical trajectory model, or the so-called three-step model, has been originally proposed to describe the HHG from noble gases $[13,32,33]$. The model properly describes key features of the HHG spectrum such as the cutoff energy. Recently, the semiclassical trajectory model was extended to the solid-state HHG [34]. In the solid-state semiclassical trajectory model, the HHG is described by the following three steps:

(1) Creation of an electron-hole pair by exciting an electron from a valence band to a conduction band at the optical gap of the solid.

(2) Acceleration of the electron-hole pair by an external laser field.

(3) Emission of a high-energy photon by the recombination of the accelerated electron-hole pair.

The major difference of the solid-state semiclassical trajectory model from the corresponding gas-phase model is the treatment of the acceleration of particles in the second step: since ionized electrons travel in vacuum in the gas-phase model, they can be treated as free charged-particles, which have a parabolic energy dispersion, $\epsilon(\boldsymbol{k})=\hbar^{2} \boldsymbol{k}^{2} / 2 m_{e}$ with the wave number $\boldsymbol{k}$. In contrast, the dynamics of electronhole pairs in solids is not generally described by the simple parabolic energy dispersion but requires more complex anharmonic dispersion reflecting the solid-state electronic band structure. Thus, the solid-state semiclassical trajectory model can be seen as a generalization of the gas-phase model by changing the electron mass $m_{e}$ to the effective electron-hole mass,

$$
\mu_{i j}=\left[\frac{\partial^{2}}{\partial k_{i} \partial k_{j}}\left(\epsilon_{c k}-\epsilon_{v \boldsymbol{k}}\right)\right]^{-1},
$$

where $\epsilon_{b \boldsymbol{k}}$ is the band dispersion of valence $(b=v)$ and conduction $(b=c)$ bands.

Based on the semiclassical trajectory model, the relative position $\boldsymbol{x}(t)$ of an electron-hole pair created at the time $t_{0}$ can be described as [34]

$$
\boldsymbol{x}(t)=\int_{t_{0}}^{t} d t^{\prime} \boldsymbol{v}\left[\boldsymbol{K}\left(t^{\prime}\right)\right],
$$

where $\boldsymbol{K}(t)=\boldsymbol{k}+\boldsymbol{A}(t)$ is the shifted wave vector due to the applied vector potential $\boldsymbol{A}(t)$ by the acceleration theorem. The relative electron-hole velocity $\boldsymbol{v}(\boldsymbol{k})$ is determined by the electron-hole energy dispersion as

$$
\boldsymbol{v}(\boldsymbol{k})=\boldsymbol{v}_{c}(\boldsymbol{k})-\boldsymbol{v}_{v}(\boldsymbol{k})=\frac{\partial}{\partial \boldsymbol{k}}\left[\epsilon_{c k}-\epsilon_{v \boldsymbol{k}}\right] .
$$

In the semiclassical trajectory model, an electron-hole pair is assumed to be created at the band gap with zero distance $\boldsymbol{x}\left(t_{0}\right)=0$. Then, the trajectory $\boldsymbol{x}(t)$ is evolved with Eq. (2). In the final step, the electron-hole pair is recombined at time $t_{r}$ and emits a photon when the electron and hole come back to the same position $\boldsymbol{x}\left(t_{r}\right)=0$. The emitted photon energy corresponds to the energy of the recombined electron-hole pair, $\epsilon_{c \boldsymbol{K}\left(t_{r}\right)}-\epsilon_{v \boldsymbol{K}\left(t_{r}\right)}$.

It is worth noting that the semiclassical trajectory model considers two kinds of trajectories: one is a trajectory in the real space with Eq. (2) while the other is that in the momentum space.

\section{B. Electron scattering effect in the semiclassical trajectory model}

In the above semiclassical model, dynamics of ionized electrons or created electron-hole pairs is treated as independent free particles. This treatment is accurate enough to describe the HHG from dilute gases. However, in solids, electrons and holes can be scattered by phonons, other electrons and holes, impurities and many other processes. Therefore, the free-particle treatment without scattering processes is not complete to describe the solid-state HHG. The importance of such scattering processes could also be seen in the recently observed multiplateau feature in HHG spectra. Unlike many HHG spectra from semiconductors featuring single plateau, the experiments using noble-gas solids [35] demonstrate the multiple plateaus feature in the HHG spectra. Furthermore, it has been demonstrated that such multiplateau feature can be theoretically described by ladder-climbing process [36], which is conceptually based on Umklapp scattering. Thus, the multiplateau feature could be seen as the consequence of the scattering effect. However, in the previous study proposing the ladder-climbing process, only the $k$-space (crystal momentum space) semiclassical trajectories have been considered, and it was assumed that electron-hole pairs can recombine at any instance of time. As a result, an electron and a hole are allowed to recombine and emit a photon no matter how far they are separated in real space. In this work, we integrate Umklapp scattering effect into the semiclassical trajectory model using both real- and $k$-space trajectories. This allows us to obtain the information of recombination time for electron-hole pairs and thereby refine the solid-state semiclassical trajectory model.

The incorporation of Umklapp scattering effect with the semiclassical trajectory model is carried out as follows:

(1) Creating an electron-hole pair at the time $t_{0}$ by exciting an electron from a valence band to a conduction band at the Bloch wave vector, $\boldsymbol{k}_{0}$ that corresponds to the optical gap of the solid.

(2) Propagating the trajectory in real-space, $\boldsymbol{x}(t)$, with Eq. (2) and the trajectory in $k$-momentum-space, $\boldsymbol{k}_{0}+\boldsymbol{A}(t)-$ $\boldsymbol{A}\left(t_{0}\right)$, with the acceleration theorem.

(3) Branching a trajectory into scattered and nonscattered trajectories when the trajectory reaches the Brillouin zone edge. Here, Umklapp scattering is described as the sudden 


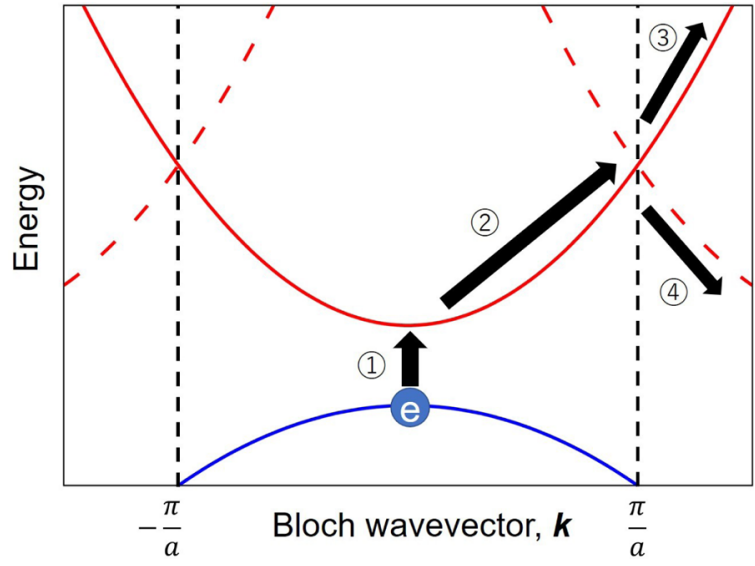

FIG. 1. Schematic picture of semiclassical trajectory dynamics and Umklapp scattering. The blue-solid (lower) line describes a valence band while the red-solid (upper) line describes an unfolded conduction band. The replicated unfolded-bands with the reciprocal lattice vector are indicated as the red-dashed lines.

shift of the accelerated Bloch vector $\boldsymbol{K}(t) \rightarrow \boldsymbol{K}(t) \pm n \boldsymbol{b}$ with the reciprocal lattice vector, $\boldsymbol{b}$ and an integer $n$. The details of the scattering is depicted in Fig. 1. After the scattering, the branched trajectories are propagated in the same way described in the step 2 .

(4) Recombining the electron-hole pair at time $t_{r}$ when $\boldsymbol{x}\left(t_{r}\right)=0$, and emitting a photon whose energy corresponds to the energy difference of the electron-hole pair, $\epsilon_{c, \boldsymbol{K}\left(t_{r}\right)}-$ $\epsilon_{v, \boldsymbol{K}\left(t_{r}\right)}$.

Figure 1 shows a schematic picture of the branching procedure discussed above. The red-solid line shows an unfolded conduction band while the blue-solid line shows a valence band. The replicated conduction bands shifted with the reciprocal lattice vector are described as the red-dashed lines. In Fig. 1, arrow (1) describes the excitation of an electron from the top of the valence band to the bottom of the conduction band. Arrow (2) indicates the acceleration of the electron from the conduction bottom to the Brillouin zone edge. At the Brillouin zone edge, the conduction bands have a crossing, or they may have an avoid-crossing. After the crossing at the Brillouin zone edge, the electron trajectory may diabatically follow the red-solid line as depicted with arrow (3) or may be switched to the red-dashed line as (4). Since the red-dashed lines are nothing but the replicated bands with the crystal momentum shift by the reciprocal lattice vector, the switching of the bands is nothing but Umklapp scattering at the Brillouin zone edge. In general, where bands are crossing, the interband transition may occur due to scattering processes. In this work, such scattering effect is incorporated with switching of the trajectory on energy bands.

\section{RESULTS}

In this section, we first examine the difference between the simple parabolic band dispersion and the nonparabolic solid-state band dispersion in the context of the semiclassical trajectory analysis. For this purpose, we employ the Kane band model [37]. Then, we elucidate the role of Umklapp scattering in the HHG by comparing the semiclassical trajectory

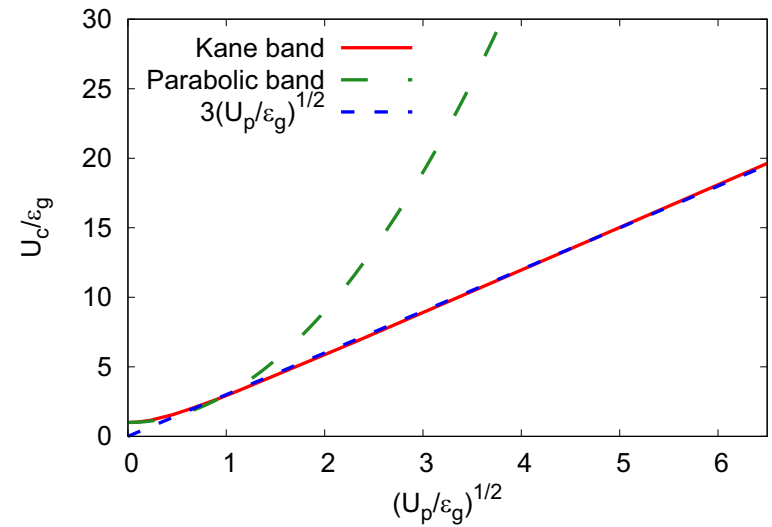

FIG. 2. Cutoff energy of the HHG $U_{c}$ as a function of applied laser field strength $F_{0}$, which is proportional to the square root of the ponderomotive energy $U_{p}$. The red-solid line shows the result of the Kane band model while the green-dashed line shows that of the parabolic band model. The blue-dotted line indicates the analytic line, $U_{c} / \epsilon_{g}=3\left(U_{p} / \epsilon_{g}\right)^{1 / 2}$.

model with the one-dimensional quantum electron dynamics simulation. Finally, we further explore the effect of more general scattering with the mean-free path approximation.

\section{A. Solid-state trajectory model without scattering}

To study the solid-state HHG with the semiclassical model, we first examine the semiclassical trajectory analysis with the Kane band model [37] without the scattering contribution. The Kane band model is widely used to model the solid-state electronic band structures. For example, the seminal work for field-induced ionization by Keldysh [38], which finds applications in many fields [39-41], is based on the Kane band.

Here, we briefly assess the difference between the parabolic band dispersion and the solid-state Kane band dispersion in the semiclassical trajectory model of HHG. The parabolic energy dispersion is described as

$$
\epsilon_{\text {parabolic }}(\boldsymbol{k})=\epsilon_{g}+\frac{|\boldsymbol{k}|^{2}}{2 \mu},
$$

where $\epsilon_{g}$ is the band gap, and $\mu$ is the electron-hole reduced mass. With the same parameters, the Kane band can be described as

$$
\epsilon_{\text {Kane }}(\boldsymbol{k})=\epsilon_{g} \sqrt{1+\frac{|\boldsymbol{k}|^{2}}{\mu \epsilon_{g}}} .
$$

According to previous works $[13,34]$ based on the semiclassical trajectory model, one can evaluate the cutoff energy of HHG as the maximum recombination energy among all possible trajectories under monochromatic laser fields. Figure 2 shows the cutoff energy $U_{c}$ of the semiclassical trajectory model as a function of the square root of the ponderomotive energy $\sqrt{U_{p}}$, which is defined as $U_{p}=F_{0}^{2} / 4 \mu \omega_{0}^{2}$ with the laser field strength $F_{0}$, the effective mass $\mu$ and the laser angular frequency $\omega_{0}$. The red-solid line shows the result of the Kane band model, while the green-dashed line shows that of the parabolic band model. For the parabolic dispersion 
case, the cutoff energy $U_{c}$ is described by the well-known formula as [13]

$$
U_{c}^{\text {(parabolic) }}=\epsilon_{g}+3.17 U_{p} .
$$

As seen from Fig. 2, in the weak field region (small $U_{p}$ region), the two models give the similar cutoff energy. This observation can be understood by a fact that the Kane band model is reduced to the parabolic band model in the small wave number limit as $\epsilon_{\text {Kane }}(\boldsymbol{k}) \rightarrow \epsilon_{g}+|\boldsymbol{k}|^{2} / 2 \mu,(|\boldsymbol{k}| \rightarrow 0)$. As seen from Fig. 2, the two models show qualitatively different behaviors in the strong field region: the cutoff energy of the Kane band model is proportional to the field strength, $F_{0} \sim$ $\sqrt{U_{p}}$, while that of the parabolic band model is proportional to the square of the field strength. The quadratic field-strength dependence of the parabolic band model is consistent with the cutoff energy of the gas-phase HHG while the linear fieldstrength dependence is consistent with the reported feature of the solid-state HHG [22-24].

In Fig. 2, the blue-dotted line shows the analytic line, $U_{c} / \epsilon_{g}=3\left(U_{p} / \epsilon_{g}\right)^{1 / 2}$. One sees that the analytic blue-dotted line shows nice agreement with the result of the Kane band model (red-solid line). Therefore, in the strong field limit, the cutoff energy of the Kane band model is well approximated as

$$
U_{c}^{\text {Kane }} \approx 3 \epsilon_{g} \sqrt{U_{p} / \epsilon_{g}}=\frac{3}{2} v_{g}^{\infty} \frac{F_{0}}{\omega_{0}},
$$

where $v_{g}^{\infty}$ is the group electron-hole velocity of the Kane band model in the large $|\boldsymbol{k}|$ limit,

$$
v_{g}^{\infty}=\lim _{|\boldsymbol{k}| \rightarrow \infty}\left|\frac{\partial}{\partial \boldsymbol{k}} \epsilon_{\text {Kane }}(\boldsymbol{k})\right|=\sqrt{\frac{\epsilon_{g}}{\mu}} .
$$

In contrast, the $k$-space semiclassical trajectory model [36] provides the following cutoff formula in the strong field limit

$$
U_{c}^{k \text {-space }}=\epsilon_{\text {Kane }}\left(\frac{2 F_{0}}{\omega_{0}}\right) \approx 2 \sqrt{\frac{\epsilon_{g}}{\mu}} \frac{F_{0}}{\omega_{0}}=2 v_{g}^{\infty} \frac{F_{0}}{\omega_{0}} .
$$

Note that the same cutoff expression has been derived for the analysis on graphene [42] with a similar consideration to the $k$-space trajectory. Comparing Eqs. (7) and (9), the real-space trajectory model provides the smaller cutoff energy than the $k$-space trajectory model by a factor of $3 / 4$. In the $k$-space trajectory model, the recombination is allowed at any instance of time. However, in the real-space trajectory model, the recombination is allowed only when paired electron and hole come to the same position. Therefore, the recombination events in the real-space trajectory model is a subset of those in the $k$-space model. Hence the cutoff energy of the realspace model is smaller than that of the $k$-space model by construction.

\section{B. Umklapp scattering contribution to the HHG}

Here, we investigate the role of Umklapp scattering in the semiclassical trajectory model. For this purpose, we compare the semiclassical trajectory model with the one-dimensional quantum mechanical (1D-QM) simulation. We employ the same model for the 1D-QM simulation as the previous work [36]. We first briefly explain the 1D-QM simulation, and then introduce the corresponding semiclassical trajectory model with Umklapp scattering.
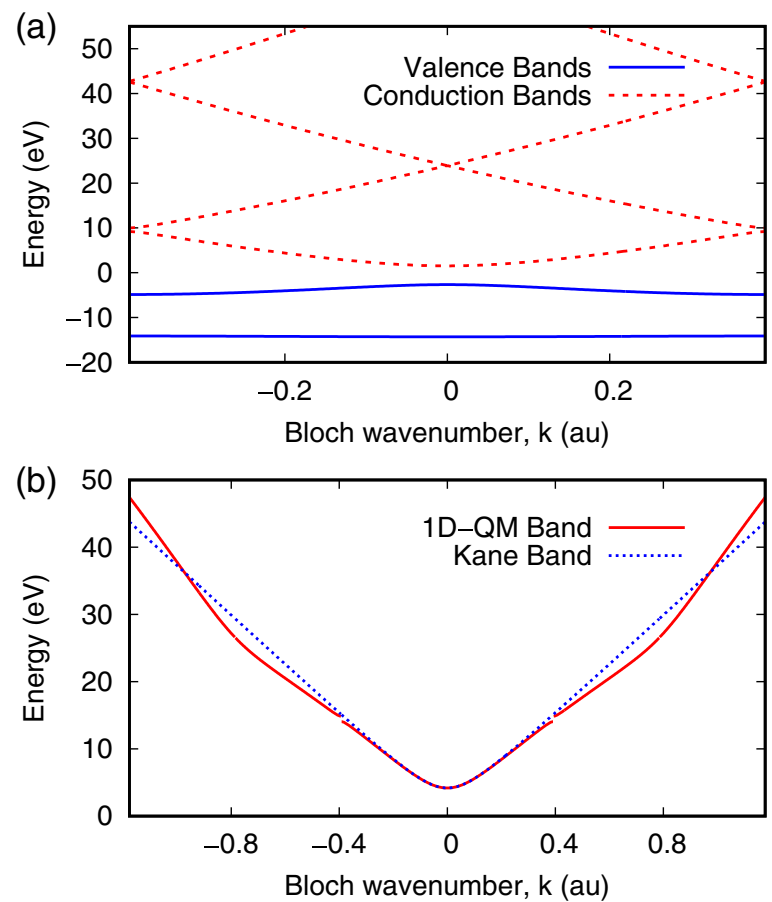

FIG. 3. (a) Electronic band structure of the one-dimensional quantum systems described by the Matthieu-type lattice potential, Eq. (11). The valence bands are shown as the blue-solid lines while the conduction bands are shown as the red-dotted lines. (b) Comparison of the Kane band (blue-dotted) and the electron-hole band (red-solid), which is defined as the energy difference between the conduction bands and the top valence band, $\epsilon_{c, k}-\epsilon_{v, k}$.

The 1D-QM system is described by the following singleparticle Schrödinger equation

$$
i \frac{\partial u_{b, k}(x, t)}{\partial t}=\left\{\frac{1}{2}\left[-i \frac{\partial}{\partial x}+k+A(t)\right]^{2}+v(x)\right\} u_{b, k}(x, t),
$$

where $u_{b, k}(x, t)$ are periodic part of the Bloch orbitals with the band index $b$ and the Bloch wave number $k$. Here, $A(t)$ is the spatially uniform time-dependent vector potential and $v(x)$ is the single-particle potential. For the single-particle potential $v(x)$, we employ the Mathieu-type lattice potential

$$
V(x)=V_{0} \cos \left(\frac{2 \pi}{L} x\right),
$$

where the potential height $V_{0}$ is set to 0.37 a.u. and the lattice constant $L$ is set to 8 a.u.

In this work, the system is discretized by grid points for both real and crystal momentum spaces. The real-space unit-cell is discretized with 30 equally spaced grid points, and the first Brillouin zone is discretized with 352 uniformly spaced grid points. For the time propagation of the timedependent Schrödigner equation we employ the Taylor expansion scheme [43] with the single time step $\Delta t$ set to 1 attosecond.

Figure 3(a) shows the computed band structure of the 1D-QM model. The bottom two bands are treated as valence bands, and all the other bands are treated as the conduction 
bands. For the comparison with the semiclassical trajectory model, we fit the band structure of the 1D-QM model by the Kane band model. Figure 3(b) shows the Kane band $\epsilon_{\text {Kane }}(k)$ and the particle-hole energy band structure of the 1D-QM model. The particle-hole energy bands are defined by the difference between the conduction bands and the top valence band as $\epsilon_{c, k}-\epsilon_{v, k}$, and they are unfolded in Fig. 3(b). Here, the effective mass $\mu$ of the Kane band model is set to $0.083 m_{e}$, and the band gap $\epsilon_{g}$ is set to $4.18 \mathrm{eV}$. These values are extracted from the 1D-QM model. Hereafter, for simplicity, we employ the Kane band model for the semiclassical trajectory simulation instead of the band structure from the 1D-QM model. However, it is worth noting that one can also employ the semiclassical trajectory model with the 1D-QM band structure by considering hopping between bands at the Brillouin zone edge as considered in the previous ladder climbing process [36].

We evaluate the HHG spectrum of the 1D-QM model by exactly solving numerically the time-dependent Schrödinger equation, Eq. (10), using the following form for the applied vector potential:

$$
A(t)=\frac{F_{0}}{\omega_{0}} \sin ^{4}\left(\frac{\pi}{\tau} t\right) \sin \left(\omega_{0} t\right),
$$

in the duration $0<t<\tau$ and zero outside. Here, $\tau$ is the full duration of the pulse. In this work, we set the mean photon energy $\hbar \omega_{0}$ to $387 \mathrm{meV}$ (with corresponding wavelength $3200 \mathrm{~nm}$ ), and the full duration of the pulse $\tau$ to 96.1 fs (equivalent to 9 periods of the mean photon frequency) according to the previous work [36].

During the time propagation, the electric current $J(t)$ can be evaluated by

$$
\begin{aligned}
J(t)= & -\sum_{b=1}^{2} \frac{1}{2 \pi} \int_{-\pi / L}^{\pi / L} d k \\
& \times \int_{0}^{L} d x u_{b k}^{*}(x, t)\left[-i \frac{\partial}{\partial x}+k+A(t)\right] u_{b, k}(x, t) .
\end{aligned}
$$

Furthermore, the HHG spectrum $I(\omega)$ can be evaluated as the Fourier transform of the current as

$$
I(\omega) \sim \omega^{2}\left|\int_{0}^{T_{\text {pulse }}} d t \sin ^{4}\left(\frac{\pi}{\tau} t\right) J(t) e^{i \omega t}\right|^{2},
$$

where the Fourier transform of the current is evaluated with the same envelope function as the applied laser pulse.

Figure 4 shows the computed power spectrum of the HHG from 1D-QM model with the field strength of $F_{0}=$ $0.165 \mathrm{~V} / \AA$, showing the clear multiple plateaus. The multiplateau feature was investigated with the $k$-space semiclassical trajectory model, and it was explained by the ladder climbing process in the band structure [36]. In this subsection, we explore the multiplateau feature based on the real-space semiclassical trajectory with the scattering effect.

In our model we explore the multiplateau feature based on both the $k$-space and the real-space semiclassical trajectory with the scattering effect. Figure 5 shows the HHG spectra as functions of the applied field strength $F_{0}$. One can see the formation of the multiplateau feature with increase of the field strength. To assess the semiclassical trajectory model,

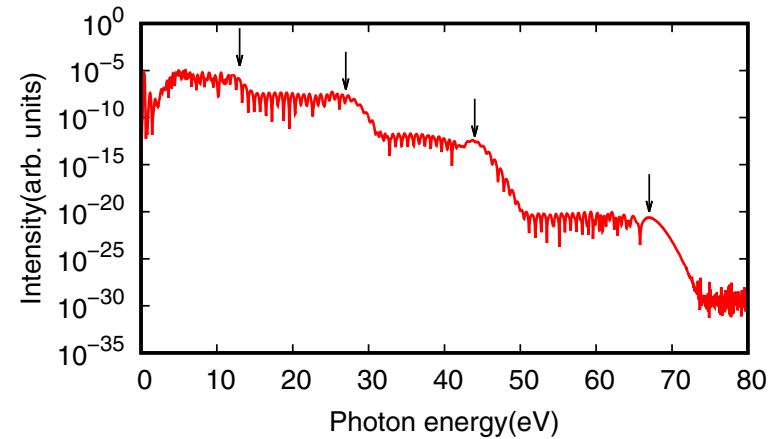

FIG. 4. HHG spectrum computed by the quantum simulation with the field strength of $F_{0}=0.165 \mathrm{~V} / \AA$. The cutoff energy of multiple plateaus are indicated by black arrows around 13, 28, 44, and $68 \mathrm{eV}$.

the computed cutoff energy with the Kane band model is also shown as the black-solid line (nonscattered), which is nothing but the red-solid line shown in Fig. 2. One sees that the first cutoff of the 1D-QM model is captured by the semiclassical trajectory model without scattering (black line).

To study the role of scattering, we evaluate the maximum recombination energy among all possible scattered trajectories as the cutoff energy of the scattered trajectory. In Fig. 5, the blue-dashed line shows the maximum recombination energy among the singly scattered trajectories, and the reddotted line shows that among the doubly scattered trajectories. By comparing the cutoff energies of the scattered trajectories with the HHG spectra of the 1D-QM model, one sees that the singly scattered trajectories (blue-dashed line) provide the second cutoff of the 1D-QM simulation while the doubly scattered trajectories(red-dotted line) provide the third cutoff. Therefore, the formation of the multiplateau feature in the

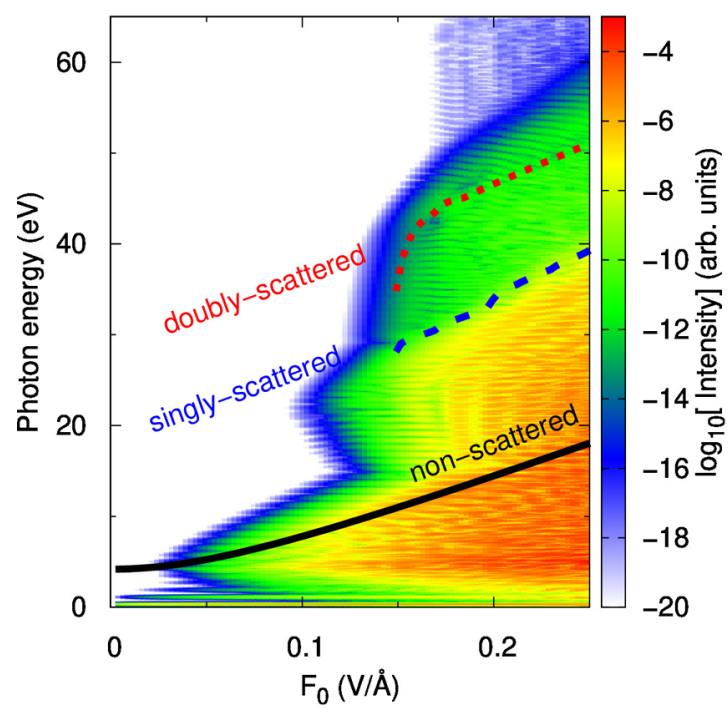

FIG. 5. Spectra of HHG computed by the 1D-QM simulation as functions of the field strength, $F_{0}$. The cutoff energies computed by the semiclassical trajectory model with the Kane band are also shown: The black-solid line shows the result without scattering, the blue-dashed line shows that of singly scattered trajectories, and the red-dotted line shows that of doubly scattered trajectories. 

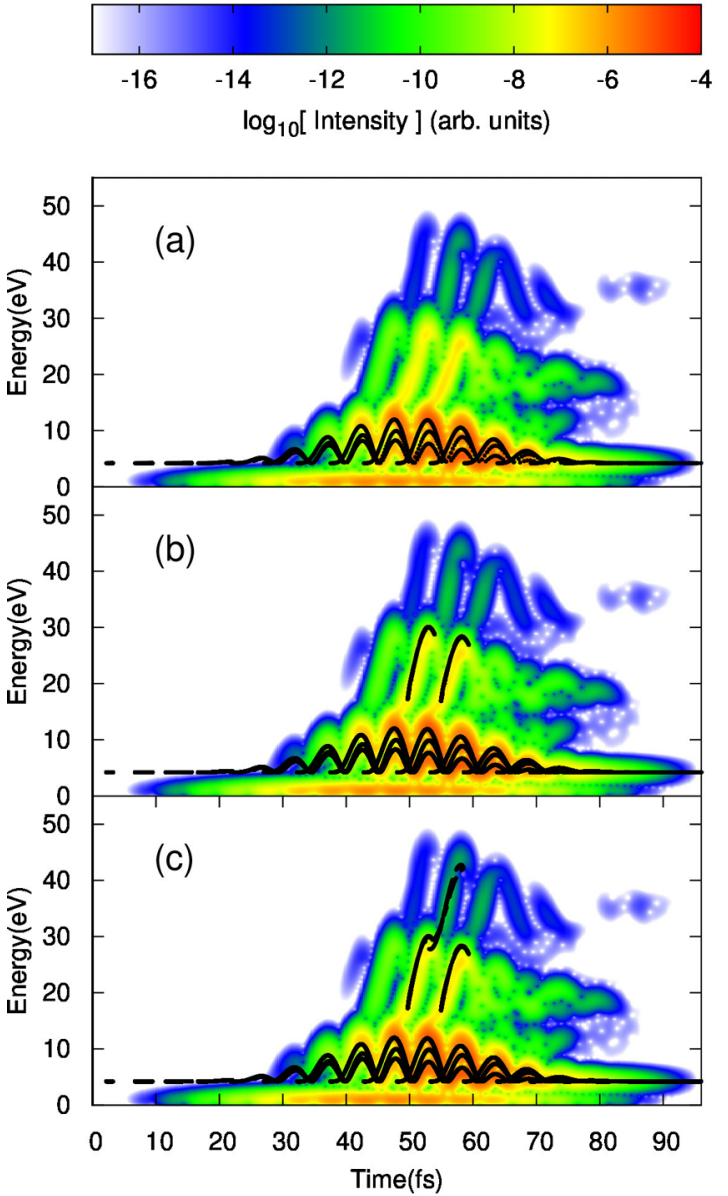

FIG. 6. Temporal evolution of the HHG computed with the 1DQM simulation. The black-dots describe the emitted photon energy and the emission timing, computed by the semiclassical trajectory model with the Kane band. Each panel shows the contribution from semiclassical trajectories with different number of scattering: (a) No scattering, (b) single scattering, and (c) double scattering.

HHG spectrum can be understood as the consequence of Umklapp scattering. This real-space scattering interpretation is a complementary picture of the previous ladder climbing picture in the $k$-space [36].

Note that, we examined the semiclassical trajectory model with the direct use of the 1D-QM band and confirmed that the result of the Kane band model shows fair agreement with that of the band structure of the 1D-QM model. Thus, the use of the simple Kane band approximation has been validated. For more details, see the discussion in the Appendix.

To study further details of the scattering effect in the HHG from solids, we elucidate the temporal structure of HHG. For this purpose, we perform the Gabor transformation to the current $J(t)$ with the time window function whose the full width of the half maximum (FWHM) is $1.78 \mathrm{fs}$. The computed temporal evolution of HHG from the 1D-QM model is shown in Fig. 6. Here, the field strength $F_{0}$ is set to 0.165 $\mathrm{V} / \AA$. Note that, in all the panels of Fig. 6, the same result of the 1D-QM model is shown. In addition to the 1D-QM result, the recombination energy and timing evaluated by the semiclassical trajectory model of different number of scatter- ing are depicted in different panels; Figs. 6(a)-6(c) show the results of nonscattered, singly scattered, and doubly scattered trajectories, respectively.

As seen from Fig. 6(a), the nonscattered trajectories contribute only to the first plateau of HHG. In contrast, in Fig. 6(b), the singly scattered trajectories show the contribution to the second plateau (around 13 to $28 \mathrm{eV}$ ). Furthermore, from Fig. 6(c), the doubly scattered trajectories contribute to the formation of the third plateau (around 28 to $44 \mathrm{eV}$ ). Therefore, Umklapp scattering processes open higher energy channels for the trajectory dynamics, resulting in the multiplateau feature as a consequence of the multiple scatterings.

In Figs. 6(a)-6(c), one sees that the real-space trajectory model fairly captures the recombination energy and timings. This is a distinct feature from the $k$-space trajectory model where the recombination timing is arbitrary and can occur any instance of time. This fact indicates the importance of the real-space trajectory picture to describe the HHG even with scattering process.

Note that the present real-space trajectory model does not capture all the features of the 1D-QM simulation. For example, in Fig. 6(c) semiclassical model fails to reproduce the signals on the left side of the classical prediction in the second plateau or the signal on the right side of the classical prediction in the third plateau. These additional features can be understood by the quantum wave-packet effect. In the present semiclassical model, the excitation occurs only at the optical gap, and the scattering takes place only at the band crossing points. However, in the quantum system, the excitation and scattering can occur with finite width in the $k$-space. Additionally, in the present model, recombination only occurs when electrons and holes meet each other at the exact same position, but the quantum wave-packet nature may relax this condition and allow for recombination for a pair at nonzero separation [44]. As a result, the full quantum systems can involve more trajectories and add the additional features to the semiclassical model. Here it is also worth pointing out that electrons and holes are assumed to be localized in real space in our model as they are treated as classical particles. However, in the previous work [36] electrons and holes are assumed to be completely delocalized and they could recombine at any time. Therefore, the two models can be seen as two extreme cases, and the reality should lie somewhere in between. As mentioned above, one of the possible routes towards more realistic description is the integration of the wave-packet effect into the real-space trajectory model, and work along these lines is already under way.

\section{Loss of trajectories by electron scattering in the HHG}

In the above analysis, we investigated the role of Umklapp scattering in one-dimensional systems. Because only the forward or backward scatterings are allowed in the one-dimensional systems, the scattered trajectory can be recombined with relatively high probability. In contrast, the contribution from scattered trajectories in two- and threedimensional systems is expected to be significantly suppressed since trajectories can be scattered into a variety of directions, thus reducing the probability of recombination for returning trajectories. In addition to the consideration on 


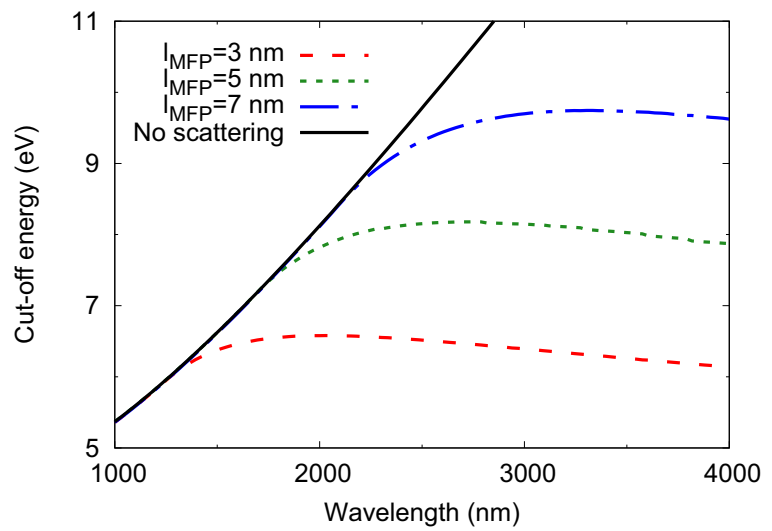

FIG. 7. Wavelength dependence of the cutoff energy of HHG, evaluated by the semiclassical trajectory model with the mean-freepath approximation. The results with several mean-free-path length $l_{\text {MFP }}$ are described. The result without scattering process is also shown as the black-solid line.

dimensionality, there are many scattering processes in solids other than Umklapp scattering. These scattering processes may also play an important role in solid-phase HHG.

To assess the impact of the suppression of HHG by various scattering processes in the higher-dimensional space, we consider a simple mean-free-path model instead of the trajectory branching mentioned above. In the mean-free-path model, we compute the trajectory length $l_{d}(t)$ as

$$
l_{d}(t)=\int_{t_{0}}^{t} d t^{\prime}\left|\boldsymbol{v}\left[\boldsymbol{K}\left(t^{\prime}\right)\right]\right| .
$$

Furthermore, we simply assume that semiclassical trajectories do not contribute to HHG anymore once the trajectory length reaches a given mean-free-path length, $l_{\mathrm{MFP}}$. Integrating this destructive contribution of scattering into the semiclassical trajectory model instead of the trajectory-branching process, we evaluate the maximum recombination energy among all trajectories before their trajectory length reaches the meanfree-path length.

Figure 7 shows the computed cutoff energy from the semiclassical trajectory model for different mean-free-path length $l_{\text {MFP }}$ as functions of the applied laser wavelength under the fixed field strength $F_{0}=0.165 \mathrm{~V} / \AA$ A. As seen from Fig. 7, the trajectory model without scattering (black-solid line) shows almost linear dependency in the long wavelength region. This behavior can be understood as the asymptotic linear dispersion of the Kane band model in the large Bloch wave vector region, as described in Eq. (7).

Once the loss of trajectories is introduced via the meanfree-path approximation, the semiclassical trajectory model shows the saturation of the HHG cutoff energy in the long wavelength region. This saturation can be understood by a fact that, even if laser fields with longer wavelength can induce higher energy trajectories, such higher-energy trajectories have longer travel distance and they are lost by the scattering process.

One sees that the cutoff energy of the trajectory model with the scattering effect in Fig. 7 becomes almost constant in the long wavelength region. This behavior is nothing but the wavelength independence of the HHG cutoff energy, and it is consistent with one of the features of the HHG from solids $[22,24,25]$. Thus, the loss of trajectories by scattering can be one of the physical mechanisms of the wavelength independence of the cutoff energy of HHG from solids. To clarify the details of the trajectory loss effect, the comparison of the trajectory model with the three-dimensional realistic simulations such as the $a b$ initio time-dependent density functional theory (TDDFT) simulation, which also captures the wavelength independence of the HHG cutoff energy [25], will be useful. It is worth noting that, although a conventional TDDFT-based simulation with approximated exchangecorrelation functionals has a lack of some scattering effects, still it can capture major contributions from elastic electronion scatterings including Umkpapp scattering. Therefore, $a b$ initio simulations based on the TDDFT would be a suitable approach for examining how Umklapp scattering contributes to HHG within larger dimensional systems. However, such analysis is beyond the scope of the present work, and will be investigated in the future.

\section{SUMMARY}

We studied the effect of electron scattering in the HHG from solids based on the semiclassical trajectory description. We first extended the solid-state semiclassical trajectory model [34] by integrating the Umklapp scattering with the Kane band model [37]. The extended model has been examined by comparing with the one-dimensional quantum dynamical simulation used in Ref. [36]. As a result, the multiplateau feature of the HHG spectra of the one-dimensional quantum model has been fairly captured by the contribution from the multiple Umklapp-scattered trajectories under the laser field acceleration. Therefore, we concluded that the multiplateau feature is the consequence of the Umklapp scattering. In the previous work based on the $k$-space trajectory model [36], the same multiplateau feature has been interpreted as the consequence of the ladder-climbing process in the electronic band structure. The two interpretations based on the real-space and $k$-space trajectories are equivalent but offers different views into the same phenomenon: the ladder-climbing process in $k$-space can be seen as the dynamics with Umklapp scattering in real-space.

We further examine the effect of Umklapp scattering by evaluating the recombination timing of electron-hole pairs and the emitted photon energy. By comparing the results of the semiclassical trajectory model with the temporal structure of the HHG spectra of the one-dimensional quantum model, we confirm that the semiclassical trajectory model with Umklapp scattering properly captures the timing and energy of the HHG from solids. This fact demonstrates that the semiclassical real-space trajectories play an important role in the microscopic mechanism of the HHG from solids because the recombination timing is not determined in the purely $k$-space trajectory model, where the recombination can occur at any time instance.

Then, we explored other consequence of the electron scattering, considering that scattered trajectories in a higherdimensional space are less likely to recombine. To take into account this effect, we evaluate the travel distance of each 
trajectory and disregard trajectories if their travel distance reaches a given mean-free-path length. We evaluated the wavelength dependence of the HHG cutoff energy by the semiclassical trajectory model with the mean-free-path approximation. As a result, we found that the cutoff energy is significantly suppressed under the longer wavelength laser driving since the longer wavelength laser field tends to induce longer travel distance for trajectories. In addition, we found that the cutoff energy is almost independent of the laser wavelength once the wavelength becomes long enough. This wavelength independence is consistent with a feature of the reported HHG from solids [22,24,25].

The above findings indicate that the real-space trajectory combined with scattering processes plays an essential role in the HHG from solids. Thus, the optical control of the realspace trajectories under scattering processes may further open a way to enhance and control the HHG from solids.

\section{ACKNOWLEDGMENTS}

This work was supported by the European Research Council (Grant No. ERC-2015-AdG694097), the Cluster of Excellence "Advanced Imaging of Matter" (AIM), and JSTCREST under Grant No. JP-MJCR16N5. S.A.S. gratefully acknowledges the fellowship from the Alexander von Humboldt Foundation.

\section{APPENDIX: COMPARISON OF VARIOUS SEMICLASSICAL MODELS}

In this work, we mainly investigated the real-space semiclassical trajectory model with the Kane band approximation. Here, to confirm the feasibility of the Kane band approximation in the comparison with the 1D-QM simulation, we compare the results in semiclassical trajectory analysis calculated based on the Kane band model and the direct use of the 1D-QM band. Furthermore, we compare the cutoff energy predicted by the present real-space semiclassical trajectory model and that by the previous momentum-space semiclassical trajectory analysis in Ref. [36]. Figure 8 shows the HHG spectra computed by the 1D-QM simulation as functions of the field strength. Here, we employed the same conditions as Fig. 5. In Fig. 8, four different semiclassical results are also shown: the black-solid line represents the

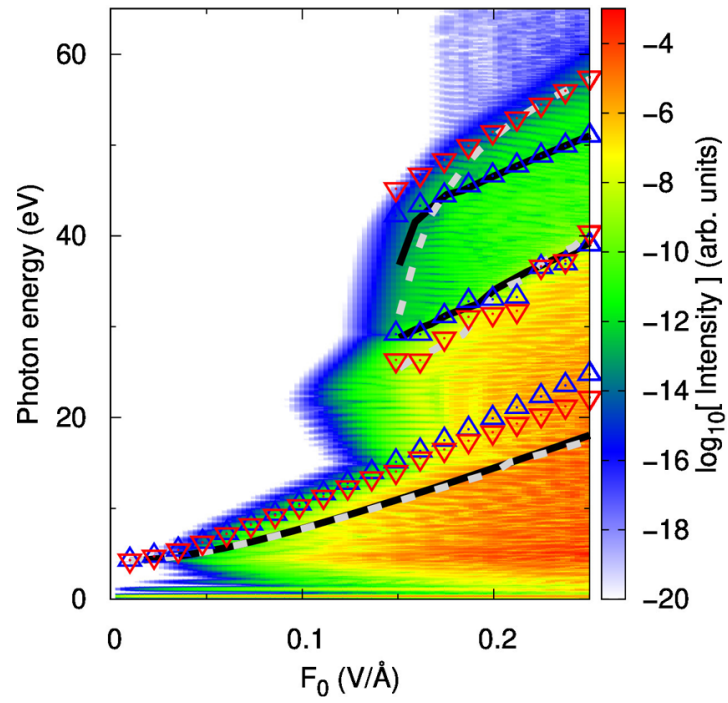

FIG. 8. Spectra of HHG computed by the 1D-QM simulation as functions of the field strength, $F_{0}$. The cutoff energies computed by the semiclassical trajectory models are also given: the black-solid line shows the result of the real-space trajectory model with the Kane band, while the gray-dashed line represents that with the 1D-QM band. The blue-upward triangles show the result of the momentumspace trajectory model with the Kane band, while the red-downwardtriangles represent that with the $1 \mathrm{D}-\mathrm{QM}$ band.

result of the real-space semiclassical trajectory model with the Kane band, and the gray-dashed line shows that of the real-space semiclassical trajectory model with the 1D-QM band. The blue-upward-triangles represent the result of the momentum space trajectory model with the Kane band, and the red-downward-triangles show that with the 1D-QM band. In general, the four cases give the very similar results, and the main multiplateau feature in the one-dimensional quantum dynamical simulation can be fairly captured by all the models.

As seen from Fig. 8, we can confirm that the Kane band and the 1D-QM band show the very similar results in the semiclassical trajectory analysis. Thus, we can clearly justify the use of the Kane band model in the semiclassical trajectory analysis for the comparison with the one-dimensional quantum simulation.
[1] G. Mourou, N. Fisch, V. Malkin, Z. Toroker, E. Khazanov, A. Sergeev, T. Tajima, and B. L. Garrec, Opt. Commun. 285, 720 (2012).

[2] F. Krausz and M. I. Stockman, Nat. Photon. 8, 205 (2014).

[3] D. N. Basov, R. D. Averitt, and D. Hsieh, Nat. Mater. 16, 1077 (2017).

[4] J. Flick, M. Ruggenthaler, H. Appel, and A. Rubio, Proc. Natl. Acad. Sci. USA 114, 3026 (2017).

[5] P. N. Butcher and D. Cotter, The Elements of Nonlinear Optics (Cambridge University Press, Cambridge, 1990), Vol. 9.

[6] R. W. Boyd, Nonlinear Optics (Academic Press, New York, 2008).

[7] M. Hentschel, R. Kienberger, C. Spielmann, G. A. Reider, N. Milosevic, T. Brabec, P. Corkum, U.
Heinzmann, M. Drescher, and F. Krausz, Nature 414, 509 (2001).

[8] T. Pfeifer, C. Spielmann, and G. Gerber, Rep. Prog. Phys. 69, 443 (2006).

[9] F. Krausz and M. Ivanov, Rev. Mod. Phys. 81, 163 (2009).

[10] P. A. Franken, A. E. Hill, C. W. Peters, and G. Weinreich, Phys. Rev. Lett. 7, 118 (1961).

[11] J. L. Krause, K. J. Schafer, and K. C. Kulander, Phys. Rev. Lett. 68, 3535 (1992).

[12] K. J. Schafer, B. Yang, L. F. DiMauro, and K. C. Kulander, Phys. Rev. Lett. 70, 1599 (1993).

[13] M. Lewenstein, P. Balcou, M. Y. Ivanov, A. L'Huillier, and P. B. Corkum, Phys. Rev. A 49, 2117 (1994). 
[14] A. McPherson, G. Gibson, H. Jara, U. Johann, T. S. Luk, I. A. McIntyre, K. Boyer, and C. K. Rhodes, J. Opt. Soc. Am. B 4, 595 (1987).

[15] M. Ferray, A. L'Huillier, X. F. Li, L. A. Lompre, G. Mainfray, and C. Manus, J. Phys. B: At. Mol. Opt. Phys. 21, L31 (1988).

[16] J. Itatani, J. Levesque, D. Zeidler, H. Niikura, H. Pépin, J. C. Kieffer, P. B. Corkum, and D. M. Villeneuve, Nature 432, 867 (2004).

[17] E. Goulielmakis, Z.-H. Loh, A. Wirth, R. Santra, N. Rohringer, V. S. Yakovlev, S. Zherebtsov, T. Pfeifer, A. M. Azzeer, M. F. Kling, S. R. Leone, and F. Krausz, Nature 466, 739 (2010).

[18] M. Schultze, K. Ramasesha, C. D. Pemmaraju, S. A. Sato, D. Whitmore, A. Gandman, J. S. Prell, L. J. Borja, D. Prendergast, K. Yabana, D. M. Neumark, and S. R. Leone, Science 346, 1348 (2014).

[19] M. Lucchini, S. A. Sato, A. Ludwig, J. Herrmann, M. Volkov, L. Kasmi, Y. Shinohara, K. Yabana, L. Gallmann, and U. Keller, Science 353, 916 (2016).

[20] M. Volkov, S. A. Sato, F. Schlaepfer, L. Kasmi, N. Hartmann, M. Lucchini, L. Gallmann, A. Rubio, and U. Keller, Nat. Phys. 15, 1145 (2019).

[21] F. Siegrist, J. A. Gessner, M. Ossiander, C. Denker, Y.-P. Chang, M. C. Schröder, A. Guggenmos, Y. Cui, J. Walowski, U. Martens, J. K. Dewhurst, U. Kleineberg, M. Münzenberg, S. Sharma, and M. Schultze, Nature 571, 240 (2019).

[22] S. Ghimire, A. D. DiChiara, E. Sistrunk, P. Agostini, L. F. DiMauro, and D. A. Reis, Nat. Phys. 7, 138 (2011).

[23] O. Schubert, M. Hohenleutner, F. Langer, B. Urbanek, C. Lange, U. Huttner, D. Golde, T. Meier, M. Kira, S. W. Koch, and R. Huber, Nat. Photon. 8, 119 (2014).

[24] T. T. Luu, M. Garg, S. Y. Kruchinin, A. Moulet, M. T. Hassan, and E. Goulielmakis, Nature 521, 498 (2015).

[25] N. Tancogne-Dejean, O. D. Mücke, F. X. Kärtner, and A. Rubio, Phys. Rev. Lett. 118, 087403 (2017).
[26] N. Yoshikawa, T. Tamaya, and K. Tanaka, Science 356, 736 (2017).

[27] N. Tancogne-Dejean, O. D. Mücke, F. X. Kärtner, and A. Rubio, Nat. Commun. 8, 745 (2017).

[28] H. Liu, Y. Li, Y. S. You, S. Ghimire, T. F. Heinz, and D. A. Reis, Nat. Phys. 13, 262 (2017).

[29] N. Tancogne-Dejean and A. Rubio, Sci. Adv. 4, eaao5207 (2018).

[30] G. Le Breton, A. Rubio, and N. Tancogne-Dejean, Phys. Rev. B 98, 165308 (2018).

[31] S. Ghimire and D. A. Reis, Nat. Phys. 15, 10 (2019).

[32] K. Kulander, K. Schafer, and J. Krause, in Super-Intense LaserAtom Physics (Springer, Berlin, 1993), pp. 95-110.

[33] P. B. Corkum, Phys. Rev. Lett. 71, 1994 (1993).

[34] G. Vampa, C. R. McDonald, G. Orlando, P. B. Corkum, and T. Brabec, Phys. Rev. B 91, 064302 (2015).

[35] G. Ndabashimiye, S. Ghimire, M. Wu, D. A. Browne, K. J. Schafer, M. B. Gaarde, and D. A. Reis, Nature 534, 520 (2016).

[36] T. Ikemachi, Y. Shinohara, T. Sato, J. Yumoto, M. KuwataGonokami, and K. L. Ishikawa, Phys. Rev. A 95, 043416 (2017).

[37] E. O. Kane, J. Phys. Chem. Solids 1, 249 (1957).

[38] L. Keldysh, Sov. Phys. JETP 20, 1307 (1965).

[39] P. Balling and J. Schou, Rep. Prog. Phys. 76, 036502 (2013).

[40] A.-C. Tien, S. Backus, H. Kapteyn, M. Murnane, and G. Mourou, Phys. Rev. Lett. 82, 3883 (1999).

[41] P. Jürgens, M. Jupé, M. Gyamfi, and D. Ristau, Proc. SPIE 10014, 100141C (2016).

[42] L. A. Chizhova, F. Libisch, and J. Burgdörfer, Phys. Rev. B 95, 085436 (2017).

[43] K. Yabana and G. F. Bertsch, Phys. Rev. B 54, 4484 (1996).

[44] L. Yue and M. B. Gaarde, Phys. Rev. Lett. 124, 153204 (2020). 